\title{
Contribuciones de la Psicología de la Liberación a la Integración de la Población Inmigrante
}

\section{Contributions of Liberation Psychology to the Integration of the Immigrant Population}

\author{
María Jesús Albar \\ Universidad de Sevilla - España \\ Manuel García-Ramírez \\ Universidad de Sevilla - España \\ Sonia Hernández-Plaza \\ Lisbon University Institute - Portugal \\ Mustapha El Karkri \\ Junta de Andalucía - España \\ Olga Paloma-Castro \\ Universidad de Cádiz - España
}

\author{
Carlos Camacho \\ Universidad de Sevilla - España \\ Rocío Garrido \\ Universidad de Huelva - España \\ Turia El Jebari \\ Asoc. de Mujeres Marroquíes Amal Andaluza - España \\ Violeta Luque-Ribelles \\ Universidad de Sevilla - España \\ Virginia Paloma \\ Universidad de Sevilla - España
}

José Manuel Sevillano

Universidad de Sevilla - España

\begin{abstract}
Resumen. La migración es una dimensión inherente a la conducta humana desde el comienzo de la humanidad. Sin embargo, los éxodos actuales de personas empobrecidas hacia las zonas más opulentas del planeta representan nuevos desafíos que necesitan ser explorados con nuevas perspectivas y aproximaciones. La psicología de la liberación puede ayudar a dar respuesta a estos desafíos. Por un lado, permite explicar el sufrimiento de personas desplazadas e inmigrantes en términos de experiencias opresivas de vida impuestas por grupos que pretenden perpetuar y aumentar sus privilegios. Por otro, permite conocer cómo los inmigrantes confrontan y superan condiciones de injusticia, destruyen su posición de oprimidos, fortalecen lazos con otros grupos y llevan a cabo acciones colectivas para asegurar cohesión social y cooperación en los contextos de recepción y logran equitativas relaciones multiculturales. Este artículo describe las iniciativas que ha llevado a cabo CESPYD (Coalición para el Estudio de la Salud, el Poder y la Diversidad) para abordar en profundidad estos aspectos. Primero, discutimos las dificultades que la perspectiva dominante de la psicología de la aculturación tiene para abordar los desafíos de las nuevas migraciones. Complementariamente, proponemos la psicología de la liberación como un adecuado enfoque para completar la perspectiva tradicional. A continuación, redefinimos los conceptos de integración y competencia cultural como procesos de empoderamiento psicopolítico y las organizaciones de base comunitaria y las organizaciones sanitarias como escenarios comunitarios empoderadores. Finalmente, este artículo propone algunas direcciones de investigación que pueden ser abordadas desde este enfoque.

Palabras clave: liberación, integración, aculturación, competencia cultural, poder, opresión, multiculturalismo.
\end{abstract}

\begin{abstract}
Mobility is a human dimension that has been a part of human behaviour since humanity began. However, the actual exodus of impoverished people to the more opulent zones of the planet represents new challenges that need to be explored with new perspectives and approaches. Liberation psychology can assist in answering these challenges. On one hand, it permits an explanation of displaced people and poor immigrants' human suffering in terms of the life experiences oppressively imposed on them by other human groups as a means to maintain privileges. On the other hand, it allows knowing how immigrants confront and overcome unjust conditions, destroy their position as oppressed, strengthen bonds with other groups, and carry out actions as a means to forge cohesion and cooperation in the contexts of reception and to achieve equitable multicultural international relations. This paper describes the initiatives that we have achieved in CESPYD for the purpose of studying these aspects in depth. First we discuss the difficulties that the dominant perspective of acculturation psychology poses in tackling the challenges that new migration represents. Additionally, we suggest liberation psychology as an adequate focus to complete a traditional perspective. Furthermore, we redefine concepts of integration and cultural competency as processes of psychopolitical empowerment and the community-based organizations and health organizations as empowering community settings. Finally, this paper proposes some research directions that can broaden its focus. Keywords: migration, liberation, integration, acculturation, cultural competence, power, oppression, multiculturalism.
\end{abstract}

La correspondencia concerniente a este artículo debe ser enviada a Manuel García-Ramírez. Facultad de Psicología. Departamento de Psicología Social. C/ Camilo José Cela, s/n. 41018-Sevilla, España. Teléfono: 34954557808.E-mail: magarcia@us.es
Este estudio ha sido en parte financiado por el Ministerio de Ciencia e Innovación (SEJ2006-14470) y por la Dirección General de Política Migratoria de la Junta de Andalucía. 
La migración ha sido parte del comportamiento humano desde el comienzo de la humanidad. Sin embargo, los éxodos actuales de gente empobrecida hacia las zonas más opulentas del planeta suponen nuevos desafíos que necesitan ser explorados con nuevas perspectivas y aproximaciones. La intensidad y singularidad de estos flujos ha hecho que las minorías etnoculturales sean parte substancial del tejido social de las comunidades receptoras. Como resultado, el debate público sobre los derechos de la población inmigrante - presentarse de acuerdo a sus creencias y valores, incluir sus pautas culturales en la vida ciudadana, disfrutar de los servicios de la comunidad, etc. - se han hecho cotidiano en los vecindarios, escuelas, servicios de salud y bienestar, medios de comunicación y en los programas de los partidos políticos. El encuentro asimétrico entre recién llegados y población local, hacen que la búsqueda de legitimidad de unos enfrente el miedo a la pérdida de estatus entre los otros, alimentando conflictos interculturales y riesgos de fragmentación social.

Para afrontar estos desafíos, la comunidad científica y los foros de decisión sobre políticas nacionales y europeas, han alertado sobre la necesidad de un análisis profundo de las fuerzas opresoras que alientan la exclusión y marginación de las poblaciones inmigrantes y animado a revisar los modelos que explican el contacto intercultural (ver Consejo de Europa http://www.coe.int/, IMISCOE http://www.imiscoe. org/; Dinh y Bond, 2008; Ward y Kagitcibasi, 2010). Tomando como punto de partida el enfoque de la psicología de la liberación (PL), la finalidad de este artículo es presentar una aproximación al estudio de la integración de la población inmigrante sobre la base de que es un proceso bidireccional, activo, ecológico y multidimensional. Por su parte la población inmigrante adquiere una nueva visión de ellos mismos, ganan capacidad para influir en las decisiones colectivas, y disfrutan de oportunidades para transformar las comunidades en las que se asientan. Al mismo tiempo, la comunidad receptora se transforma y reconoce la importancia de la pertenencia cultural, creando condiciones para la participación política y para el disfrute de ciudadanía en los recién llegados (Oliveri, 2008). Esta propuesta está basada en los estudios realizados por la Coalición para el Estudio de la Salud, el Poder y la Diversidad (CESPYD, http://ces pyd.org), formada por investigadores y agentes comunitarios y se presenta en las siguientes secciones: En primer lugar, describimos la perspectiva psicológica dominante en el estudio de las migraciones internacionales y enunciamos los principales desafíos a los que se enfrenta. Seguidamente discutiremos la utilidad de la perspectiva de la PL para responder a algunas de estas cuestiones. Por último, describimos varias propuestas de investigación orientadas a superar las condiciones de opresión que sufren la población inmigrante en Andalucía y finalmente discuti- mos la potencialidad de esta perspectiva para el avance científico del área.

\section{Migraciones Internacionales y Psicología de la Aculturación}

Tradicionalmente, la perspectiva psicológica adoptada para el estudio de las migraciones internacionales ha sido la Psicología de la Aculturación (PA, Sam y Berry, 2006), en la cual el concepto de aculturación es entendido como:

"el proceso dual de cambios culturales y psicológicos que tienen lugar como resultado del contacto entre dos o más grupos culturales y sus miembros. A nivel social, implica cambios en las estructuras sociales, en las instituciones y en las prácticas culturales. A nivel individual, implica cambios en el repertorio conductual de las personas" (Berry, 2005, p. 698-699).

En su influyente modelo, Berry (1997, 2005, 2007, 2008) entiende la integración como una de las cuatro estrategias aculturativas a través de las cuales la población inmigrante busca lograr su bienestar en función de dos dimensiones: (1) la preferencia relativa por mantener la propia identidad y cultura; y (2) la preferencia por tener contacto con otros grupos culturales. Combinado estas dos dimensiones se proponen cuatro posibles estrategias de aculturación: (1) integración, en la cual se considera valioso mantener la identidad y la cultura de origen y desarrollar relaciones con la sociedad receptora; (2) asimilación, en la que se considera valioso mantener relaciones con los grupos de la sociedad receptora; (3) separación, en la que se preservan la identidad y las características culturales, evitando relaciones con la sociedad receptora; y (4) marginación, caracterizada por la pérdida de identidad cultural y evitación con la sociedad receptora.

Paralelamente, Berry (2005) propone cuatro estrategias aculturativas en la sociedad receptora: (1) $\mathrm{mul}$ ticulturalismo, cuando la diversidad cultural es una característica aceptada y valorada en la sociedad receptora; (2) melting pot, cuando la asimilación es la opción preferida para la población inmigrante; (3) segregación, permite el mantenimiento de las características culturales de los nuevos inmigrantes, pero se rechazan las relaciones con ellos; y (4) exclusión, cuando el grupo dominante fuerza la pérdida de contacto del grupo minoritario con su cultura de origen y con los demás grupos de la sociedad receptora. Diversos estudios concluyen que la integración es una opción posible si la sociedad receptora presenta una estrategia cercana a la multiculturalidad, caracterizada por una ideología multicultural, bajos niveles de prejuicio étnico y actitudes positivas entre los diferentes grupos culturales (Berry, 1997, 2005, 2007; Besabe, Páez, Aierdi, y Jiménez-Aristizabal, 
2009; Birman, Trickett y Buchanan, 2005; Rudmin, 2006).

Esta orientación ha respaldado durante casi cuatro décadas la investigación en psicología sobre la integración de la población inmigrante en los contextos de recepción, (véanse por ejemplo, el Modelo Ampliado de Aculturación Relativa de Navas et al., 2005; o el Modelo Interactivo de Aculturación de Bourhis, Moïse, Perreault y Senécal, 1997). No obstante, presenta ciertas limitaciones para explicar y ofrecer soluciones a las migraciones internacionales actuales (ver De la Mata, García-Ramírez, Santamaría y Garrido, 2010; Rudmin, 2006; Chirkov, 2009; Ward y Masgoret, 2006). Numerosos autores han puesto de manifiesto que basar los procesos de aculturación exclusivamente en variables culturales resulta insuficiente, ya que la adaptación entre los grupos y los cambios que se derivan de ellos no son exclusivamente de naturaleza cultural. Los modelos propuestos en la PA se basan en que los individuos eligen su estrategia de aculturación; sin embargo, las relaciones de asimetría que enfrentan los recién llegados para gestionar su incorporación a la nueva sociedad, difícilmente permiten imaginar que esa elección se hace libremente. Más bien, es la posición privilegiada de la población local la que determina la aparición de ciertas formas de aculturación frente a otras-e.g., segregación y marginación (García-Ramírez, De la Mata, Paloma y Hernández-Plaza, en prensa; Rudmin, 2006). Por otra parte, aunque la aculturación es definida como un proceso dinámico, los modelos propuestos plantean una visión estática del mismo, donde la población inmigrante se presenta sometida a los vaivenes y decisiones de fuerzas superiores ajenas a ellos (Ward y Masgoret, 2006). En definitiva, a pesar de que la comunidad científica ha considerado la aculturación como un proceso bidireccional y ecológico, la mayoría de las investigaciones no explora más allá del nivel individual, depositando la responsabilidad de la integración sólo en una de las partes, la más vulnerable-los recién llegados (Rudmin, 2006).

Diferentes voces desde diversas perspectivas han sugerido que el estudio de la aculturación se enriquecería del análisis de las circunstancias contextuales en las que tiene lugar el contacto entre grupos. Se entiende que es determinante conocer el impacto de las desigualdades de poder y sus manifestaciones como el racismo, la xenofobia y la exclusión social, ya que no debe ser casual que la mayoría de la población inmigrante se asiente en barrios pobres y segregados racialmente (Orfield y Lee, 2006, cit. en Tseng y Yoshikawa, 2008). Variables tales como bienestar psicológico, sentido psicológico de comunidad y control sociopolítico son elementos que juegan un papel esencial en los procesos de aculturación (Hernández-Plaza, García-Ramírez, Camacho y Paloma, 2010; Paloma, Herrera y García-Ramírez, 2009; Tseng y Yoshikawa, 2008; Prilleltensky, 2008). Tener en cuenta estos aspectos permitiría incrementar el conocimiento sobre las migraciones, las condiciones en las que ocurren y afectan a la adaptación mutua entre inmigrantes y comunidad receptora. Por tanto, se requieren perspectivas que exploren la interdependencia entre los diferentes niveles ecológicos del fenómeno, consideren a las personas como agentes activos, con capacidad para cuestionar, resistir y transformar los contextos a lo largo de su propio proceso de aculturación (GarcíaRamírez et al., en prensa). La PL ofrece un marco relevante para abordar estos aspectos.

\section{Aculturación y Psicología de la Liberación}

La PL (Martín-Baró, 1986, 1994; Montero y Sonn, 2009; Nelson y Prilleltensky, 2005) permite abordar el fenómeno migratorio desde una perspectiva ecológica, centrada en la dinámica subyacente de poder que caracterizan las relaciones humanas. Trata de identificar, analizar y transformar las condiciones que legitiman y sustentan un determinado status quo, en el que se naturalizan relaciones asimétricas entre los grupos. $\mathrm{Su}$ visión ecológica permite definir los problemas como procesos que tienen lugar en diferentes niveles interconectados (i.e. personal, relacional y comunitario), enfatizando la interrelación dinámica entre las personas y sus sistemas sociales. Así, la inmigración es conceptualizada como un proceso de transición ecológica que implica la incorporación a un nuevo contexto sociocultural, con importantes cambios en normas y valores, la posición social de los actores implicados, sus redes de relaciones interpersonales y sus condiciones de vida (Hernández-Plaza et al., 2010). La PL aporta en los modelos de aculturación (a) la dimensión de vulnerabilidad y riesgo de exclusión de la población inmigrante en contextos de opresión y (b) cómo estos grupos desarrollan una visión crítica de las injusticias y superan las condiciones de opresión de acuerdo a sus valores y cultura (Sonn y Lewis, 2009). Es decir, la PL incorpora las dinámicas de poder en la explicación de los procesos de aculturación.

El poder es entendido en términos de capacidades y oportunidades para lograr el bienestar (Martín-Baró, 1994). Algunos grupos utilizan el poder para adquirir privilegios y establecer relaciones opresivas con otros grupos (Fanon, 1963; Martín-Baró, 1994; Nelson y Prilleltensky, 2005). La opresión en los contextos de recepción es considerada como el proceso de dominación a través del cual la población local trata de ganar y mantener privilegios sobre los recién llegados, restringiendo su acceso a los recursos y limitando su capacidad para responder (Fanon, 1963; Freire, 1972; Nelson y Prilleltensky, 2005). Estos privilegios son asegurados adoptando una ideología hegemónica etnocéntrica e institucionalizando mecanismos de control tales como la violencia, ejercida a través de restricciones de movimiento y asentamiento; explotación econó- 
mica, ejercida a través de la imposición de condiciones laborales injustas; control sexual y afectivo, negando el derecho al reagrupamiento familiar; control cultural, degradando sus valores y tradiciones culturales; control político, conculcando sus derechos al voto; $\mathrm{y}$ a través de la fragmentación social-alimentando actitudes xenófobas y racistas contra y entre los propios colectivos de inmigrantes (Moane, 2003). Privan a la población inmigrante de voz y capacidad para elegir, limitan y restringen su acceso a los recursos comunitarios y étnicos, distorsionan la información y la imagen que la población local tiene sobre ellos y sobre su cultura, estigmatizándoles y excluyéndoles de la vida ciudadana (Luque-Ribelles, 2010).

Estas consideraciones ayudan a entender que las dinámicas de las diferencias culturales, no son suficientes para promover políticas y prácticas integradoras hacia la población inmigrante, y que es importante incluir en la ecuación el peso de las condiciones sociales y políticas en las que el encuentro intergrupal tiene lugar. La inclusión de estos mecanismos de opresión en la explicación de las estrategias de aculturación, revela que a pesar del reconocimiento explícito de multiculturalismo, la segregación y la exclusión sonde hecho-estrategias aculturativas adoptadas por las sociedades receptoras. Desde la perspectiva de la población inmigrante, la vida en condiciones de opresión conlleva experiencias diarias de violencia, pobreza y miedo que conducen a una visión del self desvalorizada, que asume la desigualdad como natural. Esto puede explicar porqué las sociedades que legitiman la segregación y la exclusión de la población inmigrante, favorecen estrategias de asimilación, separación y marginación en los recién llegados (García Ramírez, et al., en prensa; García-Ramírez, Martínez, Albar y Santolaya, 2002; Hernández-Plaza, García-Ramírez, Herrera, Luque y Paloma, 2009; Hernández-Plaza, Pozo, Alonso y Martos, 2005).

Al mismo tiempo, la PL permite explicar cómo los grupos sociales que viven en condiciones de opresión, resisten y desarrollan fortalezas, afirmando que todo grupo humano tiene la capacidad para oponerse a las asimetrías de poder y transformar estructuras sociopolíticas opresivas. Estrategias aculturativas como la separación y marginación en la población inmigrante -y la adopción de una ideología multicultural por parte de grupos de la población local, representan la capacidad de las comunidades para reconstruirse de acuerdo a sus intereses, valores y necesidades. Consecuentemente, la integración aculturativa en la población inmigrante y la adopción de una visión multicultural en la población local implica un proceso de construcción del self unido a la capacidad de (a) crear significados y actuar con intencionalidad; (b) mostrar capacidad para pensar sobre uno mismo y la sociedad como un todo; y (c) mantener la cultura de herencia como sistema simbólico necesario para dar sentido a los nuevos encuentros (De la Mata y Cubero, 2003). Colectivamente, todo ello implica una ideología multicultural que reconoce la importancia de la pertenencia cultural, en la cual todos los grupos son fortalecidos, creando condiciones para la participación política y consiguiendo completa ciudadanía, repeliendo la injusticia social y el descrédito cultural en todos los ámbitos ciudadanos.

Estos supuestos permiten redefinir la psicología de la aculturación. Desde la perspectiva del recién llegado, observa la estrategia de la aculturación integración como un proceso empoderador de construcción del self. Desde la perspectiva de la población autóctona, observa el multiculturalismo como la capacidad para dotar de competencia multicultural a los colectivos e instituciones, e implicarse en la transformación de las estructuras sociales. A continuación, resumiremos algunas de las líneas de investigación que nos están permitiendo desarrollar este enfoque.

\section{La Perspectiva de la Población Inmigrante: Construyendo Integración}

La investigación transcultural ha mostrado el papel predictivo de las variables personales y grupales en el proceso de aculturación integración (Navas, et al., 2005). La edad, el nivel educativo y el género, han sido señalados como factores que establecen diferencias en el proceso de adaptación (Martínez, García Ramírez y Maya, 2001). Otras características asociadas a rasgos de diversidad cultural tales como la zona geográfica de procedencia, etnia y religión, muestran su papel diferenciador en las oportunidades que la población inmigrante disfruta para incorporarse al nuevo contexto. También se ha comprobado la capacidad predictiva de determinadas variables vinculadas al status migratorio. La situación legal es determinante para el acceso al empleo y otros recursos comunitarios; los años de estancia en el desarrollo de adaptación cultural y arraigo al contexto comunitario, y el reagrupamiento familiar, en la configuración de la red social (GarcíaRamírez, et al., 2002).

En cambio, menos atención ha recibido el análisis de las condiciones de los enclaves o áreas de asentamiento a pesar del importante papel que pueden llegar a tener en los procesos de adaptación (Portes y Rumbaut, 1996). Por ejemplo, recientemente Birman, Trickett y Buchanan (2005) encontraron entre adolescentes judíos procedentes de la antigua Unión Soviética que la identificación como judío y la república de procedencia predecían una identidad aculturativa americana en función de las características del contexto comunitario de asentamiento (enclaves étnicos concentrados vs. dispersos). Sin embargo, son necesarios estudios específicos que analicen el impacto de las condiciones de opresión en los contextos de asentamiento, ya que pueden - al interiorizar la opresiónser la causa de que la población inmigrante adopte una dinámica psicológica de subordinación. 
El control psicopolítico representa la dinámica psicológica opuesta a la opresión interiorizada y es definido como la capacidad de participar en acciones colectivas, influir en las decisiones políticas, asumir roles de liderazgo, organizar a los miembros de la comunidad, y promover cambios efectivos en los sistemas sociales y políticos (Zimmerman y Zahniser, 1991). Bajo condiciones de opresión, el desarrollo de control psicopolítico puede desempeñar un papel clave durante el proceso de aculturación, facilitando la integración a través de la participación activa en movimientos de resistencia y acciones colectivas. A continuación, detallamos el proceso de empoderamiento psicopolítico que supone la construcción de aculturación integración en contextos opresivos y el papel que juegan las asociaciones de inmigrantes como escenarios comunitarios empoderadores.

\section{Aculturación Integración como Proceso de Empoderamiento Psicopolítico}

En otro lugar (García-Ramírez, et al., en prensa) hemos propuesto entender la integración-aculturativa como un proceso por el cual los recién llegados llegan a ser una parte aceptada de la nueva sociedad a través del cual adquieren pensamiento crítico sobre las condiciones injustas, ganan capacidades para confrontar esas desigualdades y llevan a cabo acciones efectivas para superarlas. Durante este proceso, la población inmigrante transforma su identidad al tiempo que transforma los contextos donde está asentada. Esta dinámica contribuye a la construcción de una sociedad multicultural en la que todos tienen "el derecho para vivir bien en base a su propia especificidad cultural, dentro de un marco que ofrece igualdad y oportunidades reales, y una cultural democrática que permite el respeto mutuo, la negociación en caso de conflicto y el desarrollo continuo de los modelos culturales iniciales" (Oliveri, 2008, p. 35).

Estas asunciones nos permiten ver la aculturación integración como un viaje liberador hacia la plena ciudadanía, un proceso de empoderamiento basado en la adquisición de derechos y responsabilidades para ser miembros activos - políticamente capaces - de contribuir al desarrollo de la nueva sociedad (Montero y Sonn, 2009). A nivel intrapersonal, está relacionado con el desarrollo de pensamiento crítico a través de la reflexión y la evaluación. Este pensamiento crítico dirige a los individuos a construir fortalezas y creer que las condiciones pueden ser cambiadas porque no son-por naturaleza - como parecen ser en un contexto histórico específico. A nivel interpersonal, el proceso de aculturación liberador está asociado con la implicación y adquisición de nuevos lazos, organizaciones y redes sociales que desarrollan nuevos referentes sociales, incrementa los recursos de la población inmigrante y su capacidad para responder a las injusticias.
Finalmente, este proceso dirige a la implicación en acciones cívicas orientadas a la construcción de contextos sociales justos. En su conjunto, este proceso implica la reconstrucción dialógica y dual del self y de los contextos de asentamientos; a nivel ciudadano, de la exclusión a la pertenencia, a nivel interpersonal, del aislamiento a la participación y a nivel intrapersonal, desde la indefensión al bienestar (García-Ramírez et al., en prensa). En definitiva, aculturación integración es poder para ganar bienestar; es acceso igualitario, aceptación y disfrute de servicios comunitarios multiculturales, es apoyo social y oportunidades para participar, compromiso y responsabilidad mutua; es bienestar psicológico, control, competencia y autonomía.

\section{Asociaciones como Escenarios Comunitarios Empoderadores}

Las redes sociales juegan un papel clave en los procesos de aculturación integración, porque permiten al colectivo ganar capacidad de respuesta como agentes activos de la comunidad. Además, la participación en organizaciones como sindicatos, asociaciones vecinales o de inmigrantes juega un papel clave para el desarrollo de movimientos de resistencia y de acción social orientadas a lograr una distribución más equitativa del poder y los recursos (Hernández-Plaza, Alonso y Pozo, 2006; Hernández-Plaza et al., 2009; Paloma, García-Ramírez, De la Mata y Amal, 2010). En un reciente estudio (Paloma et al., 2010) hemos definimos las organizaciones de base inmigrante como escenarios comunitarios empoderadores (Maton, 2008). Así, son consideradas herramientas útiles que promueven cambios a diferentes niveles: reconstruyen la identidad de las personas activistas, mejoran la comunidad donde queda inserta, y promueven cambios a nivel estructural. Maton (2008) sistematiza seis características de los escenarios que promoverían tales cambios: (1) un sistema de creencias común; (2) actividades planificadas para lograr las metas; (3) medio relacional; (4) una clara estructura de roles; (5) existencia de individuos centrales o líderes; y (6) mecanismos organizacionales que aseguren el mantenimiento y adaptación a los cambios del contexto. En el estudio citado, describimos la experiencia de la asociación Amal-Andaluza, una organización de base comunitaria que tiene como principal objetivo facilitar la integración a las mujeres marroquíes. A través del análisis de las narrativas de mujeres activistas, usuarias, trabajadores de la comunidad, y políticos vinculados a la población inmigrante, así como las propias narrativas organizacionales pudimos comprobar que participar como activistas en Amal, favorece el desarrollo de pensamiento crítico acerca de su situación de injusticia, la adquisición de mayor capacidad y habilidad para enfrentarse a los problemas e implicarse en acciones-junto a otras mujeres - para mejorar las condiciones sociales. Por 
otro lado, las actividades realizadas por Amal propiciaron la mejora de la comunidad porque facilitaron las oportunidades laborales y de vivienda para el colectivo inmigrante, la prevención del racismo, la mejora de las relaciones interculturales, y la promoción de la diversidad como un valor. Finalmente, Amal ayuda a la promoción de una sociedad multicultural justa, a través de su participación en el Foro Andaluz de la Inmigración para representar en el gobierno andaluz los intereses pro-inmigrantes, en la Escuela Andaluza de Salud Pública, y en el comité europeo para la Defensa de los Derechos de las Mujeres Marroquíes.

En relación a los debates teóricos en aculturación, esta experiencia enseña que es posible perseguir la integración en sociedades donde los recién llegados encuentran relaciones de poder asimétricas. La participación del colectivo inmigrante a través de escenarios comunitarios empoderadores como Amal, le ayuda a resistir las condiciones sociales de opresión y llegar a ser agentes activos de su propio proceso de construcción, rompiendo el ciclo de opresión. Este trabajo es una llamada para promover un rol esencial de las organizaciones de inmigrantes en las sociedades receptoras, y para destacar la importancia de que la población inmigrante participe activamente a través de coaliciones y otras estructuras del entramado social. De esta forma, se fomentará la identificación del colectivo inmigrante con las instituciones comunitarias y estructuras sociales, consolidando el bienestar y la cohesión social. Sin embargo, no deberíamos considerar que la responsabilidad para la integración es exclusivamente de la población inmigrante; lejos de esto, la transformación social requiere la implicación de la sociedad receptora. En la próxima sección abordaremos cómo estamos abordando estos desafíos.

\section{La Perspectiva de la Sociedad Receptora: Construyendo Multiculturalismo}

Portes y Rumbaut (1996) han diferenciado los contextos de asentamientos en receptivos y excluyentes, caracterizándolos por la disponibilidad de acceder a la vivienda, oportunidades para disfrutar de los servicios comunitarios y por las dinámicas intergrupales con la población autóctona. En los contextos excluyentes u opresores el acceso a la vivienda está relacionado con las condiciones de segregación y aislamiento en los barrios, dificultades para acceder y disfrutar de los servicios comunitarios (Hernández-Plaza, Pozo y Alonso, 2004; Perkins, Florin, Rich, Wandersman y Chavis, 1990). Estas condiciones vecinales - que han sido relacionadas con un rendimiento psicológico desordenado (Wandersman y Nation, 1998; MacMillan y Chavis, 1986; Perkins y Taylor, 1996) -, pueden ser asociadas a las estrategias aculturativas marginación y separación (García-Ramírez et al., en prensa). Basándonos en las proposiciones antes descritas de la PL, hemos rea- lizado dos estudios con el fin de conocer (a) si las condiciones opresoras del contexto comunitario influyen en la relación entre adaptación cultural y adaptación psicológica-es decir, las dos variables que determinan la elección de estrategia aculturativa; y (b) qué variables del contexto comunitario están relacionadas con la integración.

Un primer estudio (Hernández-Plaza et al., 2010) exploró el supuesto de relación positiva entre la adaptación cultural y la adaptación psicológica, teniendo en cuenta que las condiciones opresivas del contexto local podían reducir las oportunidades de la población inmigrante para lograr bienestar. Se midió la competencia lingüística (adaptación cultural), la satisfacción con la vida (adaptación psicológica), y la percepción de discriminación y disponibilidad de apoyo social (factores contextuales). La muestra estuvo compuesta por 298 inmigrantes marroquíes residentes en los 12 contextos de Andalucía con mayor presencia de marroquíes en el momento de la recogida de datos. Empleando HLM, los resultados mostraron que (1) una mayor percepción de discriminación atenuaba la relación positiva entre la competencia lingüística y la satisfacción con la vida, e incluso revertía dicha relación; y (2) el apoyo social modera la relación entre la adaptación cultural y el bienestar. Por tanto, contextos locales caracterizados por altos niveles de discriminación pueden ofrecer pocas oportunidades al colectivo inmigrante para lograr bienestar, puesto que la discriminación étnica aparece como una fuerte barrera para el contacto positivo con la población autóctona y el acceso a los recursos comunitarios (García-Ramírez y Camacho, 2005; Hernández-Plaza, 2003; Martínez, García-Ramírez, Maya, Rodríguez y Checa, 1996; Navas et al., 2005; Rueda y Navas, 1996).

A partir de estos resultados, diseñamos un segundo estudio para explorar las características del contexto local que facilitan la integración comunitaria de la población inmigrante marroquí (Garrido, 2010). Mediante un análisis de clusters, marroquíes de treinta contextos locales-urbanos y rurales-de Andalucía fueron agrupados en las cuatro estrategias aculturativas (i.e. asimilación, integración, separación, marginación, Berry, 2005) Se incluyeron variables tales como la satisfacción con la vida, desarrollo sociopolítico, satisfacción con el barrio, percepción de rechazo, empleo del español, aprendizaje de costumbres españolas, grado de identidad como español/a, y grado de identidad como marroquí. Posteriormente, se realizó un análisis de regresión logística para identificar un modelo predictor del contexto local que facilitaba la aculturación integración. Los resultados mostraron que la probabilidad de que la población inmigrante esté integrada disminuye en contextos donde existen escasas emociones positivas en la población autóctona hacia la inmigrante y donde la mayoría de vecinos son inmigrantes. También disminuyen las posibilidades de vivir integrado en aquellos contextos donde hay limi- 
taciones para acceder a los servicios comunitarios y menor calidad en ellos, medidos en términos de competencia cultural de sus profesionales y sensibilidad a la diversidad. Estos hallazgos nos condujeron a diseñar estudios que explorasen la competencia cultural de los profesionales y la sensibilidad de los servicios comunitarios como indicadores de multiculturalismo justo.

\section{Competencia Cultural en los Profesionales de la Comunidad}

La integración de la población inmigrante depende en buena medida de las oportunidades que encuentran en las sociedades receptoras para disfrutar de los servicios públicos, por lo que uno de los objetivos prioritarios en las agendas internacionales de intervención consiste en asegurar la titularidad del acceso a los recursos en igualdad de condiciones que la población autóctona. Para afrontar estos desafíos, los profesionales necesitan liderar y promover servicios comunitarios sensibles a la diversidad social, que honren las diferencias culturales y construyan afinidades, relaciones sociales igualitarias y justicia social (Ingleby, Chimienti, Hatziprokopiou, Ormond, y De Freitas, 2005; Penninx Berger y Kraal, 2006; Snowden, 2005).

En esta dirección, el concepto competencia cultural han sido usado en diferentes períodos y ámbitos, convirtiéndose - a la postre - en un término clave para las comunidades de investigadores implicados en el bienestar de la población inmigrante (Fostier, 2010). Pero, a pesar del acuerdo de que un cierto grado de entendimiento, valoración y respeto por las diferencias culturales son esenciales en los profesionales de los servicios comunitarios, la falta de una conceptualización suficientemente aceptada y testada ha provocado importantes controversias acerca de sus asunciones y efectos (Sue, Zane, Nagayama y Berger, 2009). En definitiva, se carecen de modelos integrales que permitan a los proveedores de los servicios a la comunidad (a) documentar los efectos que las asimetrías y desigualdades en el acceso a los recursos comunitarios tienen en la población inmigrante; (b) ayudarles a afrontar estos efectos, promoviendo sus fortalezas para resistir y-al tiempo-promover cambios estructurales para prevenirlos; (c) establecer alianzas para el mejoramiento comunitario; (d) diseminar efectivamente los hallazgos de investigación; y (e) desarrollar políticas sociales multiculturales justas (Dana y Allen, 2008).

La psicología de la liberación (PL) permite responder a algunos de estos desafíos. Por un lado, explica cómo profesionales etnocéntricos perpetúan condiciones de contacto intergrupal que incrementan el riesgo de vulnerabilidad y exclusión de la población inmigrante. Las evidencias muestran cómo derechos explícitos sobre el acceso y disfrute de la población inmigrante de los servicios comunitarios coexisten con una probada discriminación por parte de los profesionales, retraso en los servicios, negación de prestaciones o cargas inapropiadas (Fostier, 2010; Vearey, 2008). Por otro lado, la PL permite explicitar cómo los profesionales pueden desarrollar una visión crítica de sus limitaciones para responder a las necesidades de los nuevos ciudadanos, humanizar su trato con la población inmigrante y adquirir un mayor sentido de realización profesional (Albar, 2009; García-Ramírez et al., en prensa; Sonn y Lewis, 2009). Ser culturalmente competente implica adquirir habilidades, conocimientos y actitudes para funcionar eficazmente en una sociedad plural y democrática, e identificar las circunstancias que rodean y definen a cada individuo en particular, maximizando su desarrollo óptimo y el de los sistema en el que éstos se circunscriben (Kohen, 2006; Domenig, 2008; Sue, 2001). Este es un proceso que genera cambios en los dominios cognitivo, interpersonal y colectivo de los profesionales.

A nivel cognitivo, este proceso está relacionado con el desarrollo de pensamiento crítico a través de la reflexión y evaluación. Pensamiento crítico que dirige a los profesionales a desarrollar una paulatina descodificación de su mundo, captar mecanismos que deshumanizan su actividad profesional, superar creencias que mitifican narrativas de las situaciones como naturales e inamovibles, y abrir horizontes hacia nuevos interpretaciones, que implican nuevas tareas y exigencias; es decir, hacia una nueva identidad profesional. A nivel interpersonal, está asociado a la génesis y construcción de nuevos roles profesionales en los que el trabajo colaborativo es un crucial requerimiento. El profesional comunitario debe asumir una serie de roles claves: (1) como facilitador e instigador del cambio, basado en el desarrollo de conciencia acerca de las oportunidades, derechos y recursos disponibles para resistir la opresión, facilitar cambios sociales y logar bienestar; (2) como mediadores, a través de la promoción de la participación social y relaciones positivas con la población inmigrante y otros grupos comunitarios, permitiéndoles ganar capacidad de respuesta y (3) como abogados, apoyando y liderando acciones colectivas dirigidas a lograr derechos de ciudadanía y fomentando la justicia social (Balcázar, Garate-Serafini, y Keys, 2004). A nivel colectivo, implica tener capacidad para movilizar los servicios comunitarios en el fomento de una ideología multicultural, liderar procesos de cambio, diseñar y establecer alianzas con los líderes comunitarios, reconocer a los usuarios como agentes políticos activos, ofreciendo y creando oportunidades para el desarrollo de su ciudadanía y la comunidad.

Con la finalidad de confirmar esta aproximación a la competencia cultural comunitaria, diseñamos un estudio que incluía el desarrollo de un nuevo instrumento que permita medir la estructura teórica propuesta (Sevillano, 2010). La elaboración de esta herramienta responde a la necesidad de una definición holística de 
la competencia cultural que además de las características inherentes al profesional y sus habilidades en interacción con usuarios multiculturales, también incluya las características contextuales que median en la prestación de los servicios, y dirigen hacia la gestación de estrategias aculturativas integradoras y transformadoras de los contextos comunitarios. Este instrumento, compuesto por 57 ítems valorados a través de una escala Likert de seis opciones de respuesta, está diseñado para evaluar la competencia cultural desde la perspectiva de la PL, por lo que sus subescalas se corresponden con los constructos definitorios del proceso de liberación: (a) pensamiento crítico, (b) ganar capacidad para desplegar nuevos roles profesionales y (c) la implicación en acciones transformativas. El instrumento fue aplicado a 424 profesionales de los diferentes servicios en 24 unidades territoriales de Huelva, Sevilla y Almería. Las adecuadas características psicométricas obtenidas - (a) $\alpha=.878$, (b) $\alpha=.777$ y (c) $\alpha=.876$-permiten recomendar su aplicación. A partir del trabajo desarrollado en este proyecto se abren nuevas líneas de investigación, tales como estudiar la relación entre el constructo de competencia cultural y medidas relacionadas con la calidad en el trabajo, el síndrome de burnout, entre otros. Así mismo también se abren nuevas perspectivas en el entrenamiento de la competencia cultural.

En este sentido, entre los profesionales del servicio de urgencias de Hospital Virgen Macarena de Sevilla se está llevando a cabo un proceso de capacitación en competencia cultural entre enfermeras utilizando metodología de aprendizaje dialógico, basado en la premisa de Freire (1972) de que somos seres de transformación y no de adaptación (Albar y Bochinno, 2010). Se persigue no sólo la transformación a nivel individual sino también la del contexto dónde desarrolla la enfermera su trabajo. La primera etapa del entrenamiento está dirigida a crear conciencia crítica, mediante la reflexión y evaluación de las actitudes etnocéntricas que guían la práctica enfermera, utilizando para ello estímulos, tales como dramatizaciones de situaciones de la práctica habitual, análisis de casos, de narrativas de usuarios, etc. Posteriormente, una vez creada una masa crítica en las unidades de trabajo, los participantes adquieren el compromiso de sensibilizar con sus actuaciones a otros compañeros; capacidad de respuesta y de toma de acción para transformar el contexto. A nivel interpersonal, se evidencia la necesidad de crear vínculos con otros niveles asistenciales de salud, asociaciones de inmigrantes, ámbito académico, etc., para conjuntamente construir contextos sanitarios sensibles culturalmente, sobre la base del diálogo y el consenso. Enseñar sobre estas bases supone que el papel de los formadores no es de autoridad sino de mediadores, facilitadores e instigadores del cambio y además, es una manera de generar más solidaridad entre las personas implicadas en este proceso de transformación de los contextos. Este modelo de capacita- ción puede ser empleado en profesionales de otros servicios comunitarios para fomentar sensibilidad a la diversidad.

\section{Sensibilidad a la Diversidad en los Servicios Comunitarios}

Los servicios comunitarios (i.e. escuela, servicios sociales y de salud) deben ser accesibles y pertinentes, para lo cual tienen que adaptar sus agendas y programas a las nuevas necesidades de los nuevos ciudadanos (Ingleby, 2006). Aunque en España el derecho a la asistencia sanitaria, a los servicios sociales y a la educación son universales, diferentes estudios demuestran que la población inmigrante tienen dificultades para acceder a ellos. Entre los motivos a los que se atribuyen estos hecho destacan: (1) incompatibilidad horaria de estos servicios con el trabajo, (2) exclusión social y segregación espacial en barrios con escasos recursos de la comunidad o las zonas aisladas sin acceso al transporte público, (3) dificultades con el idioma, (4) percepción de prejuicio étnico y la falta de competencia cultural en los profesionales de intervención social, (5) conocimiento insuficiente acerca de los programas y servicios disponibles, (6) baja eficacia percibida, (7) burocratización y los problemas para generar un clima apropiado para la expresión y comprensión de las necesidades del colectivo inmigrante, y (8) falta de confianza, sobre todo en el caso de inmigrantes ilegales (Hernández-Plaza, 2003; HernándezPlaza et al., 2004, 2006; Martínez et al., 1996). Estos factores se relacionan con peores resultados en salud (Martínez, García-Ramírez y Maya, 2001), bajo rendimiento en las escuelas (Soriano-Ayala, 2002) y escasa participación en organizaciones sociales (Gar-cía-Ramírez et al., 2002; Hernández-Plaza, 2003).

Para afrontar estas circunstancias y específicamente en el ámbito de los servicios de salud, hemos propuesto considerar las organizaciones sanitarias como Escenarios de Cuidados Multiculturales Empoderadores (ECME; Paloma-Castro, 2010). Los ECME quedan definidos por (1) la presencia de un sistema de creencias y valores sensibles a la diversidad, (2) unos servicios y prácticas adaptadas culturalmente y (3) un equipo de gestores y profesionales sanitarios comprometidos con la diversidad.

El sistema de creencias y valores se concretiza en una misión organizativa que reconoce la diversidad y la igualdad como referente en la prestación de cuidados, que tiene en cuenta las necesidades individuales en función del contexto socio-cultural y establece como estímulo para el desarrollo del conocimiento las necesidades expresadas por la población. Para tal fin establece contacto con otros grupos o instituciones para favorecer el intercambio entre las comunidades inmigrantes y los profesionales sanitarios, involucrán- 
dose ambos colectivos de forma simétrica en la toma de decisiones. Del mismo modo, este tipo de organizaciones se caracteriza por tener unos servicios y prácticas capaces de adaptarse a la diversidad, facilitando el acceso en igualdad de condiciones a usuarios de diferentes culturas. Por último, es imprescindible en estas organizaciones la presencia de gestores y otros profesionales comprometidos con la diversidad, dispuestos a asumir nuevos retos y a liderar acciones para el cambio.

En este sentido, la pertenencia a ECME va a facilitar que los profesionales sanitarios adquieran competencia cultural, es decir, habilidades, conocimientos y actitudes para funcionar eficazmente en una sociedad plural e identificar las circunstancias que rodean a cada individuo en particular, maximizando su desarrollo óptimo, contribuyendo a la equidad, accesibilidad, disponibilidad y calidad de los servicios (Domenig, 2008; Sue, 2001). Por otro lado, los ECME van a influir en la promoción de comunidades multiculturales saludables, que estarían definidas por considerar la diversidad como un valor, la presencia de usuarios multiculturales alfabetizados en salud, de actividades o procesos de promoción de la salud e intercambio cultural, de organizaciones multiculturales activas (voluntarios, organizaciones de autoayuda, de consumidores, etc.) e instituciones comprometidas con la equidad en salud y la justicia social (ayuntamientos, escuelas, empresas, etc.).

\section{Discusión}

La propuesta que hemos descrito ilustra el potencial de la psicología de la liberación para ofrecer una nueva perspectiva del estudio de la aculturación. Impulsa la búsqueda de modelos y perspectivas teóricas, y políticas sociales orientadas a promover el bienestar de todos los ciudadanos, independientemente de su procedencia.

Estos modelos deben proveer estrategias que permitan integrar herencia cultural, valores de justicia social, tolerancia y respeto a los derechos humanos.

Con esta perspectiva, modelos teóricos y políticas sociales deben tener en cuenta la visión del mundo de todos los individuos y grupos, especialmente la de los más vulnerables, que son también los más silenciados.

En este artículo, hemos expuesto algunos resultados e iniciativas en curso que han mostrado la necesidad y conveniencia de examinar las relaciones de poder que se establecen entre los grupos y su influencia sobre el bienestar e integración de la población inmigrante. Un aspecto esencial de los actuales éxodos migratorios es que tienen lugar en condiciones de desventaja y, -en consecuencia, obviar el papel del poder y opresión en los grupos en los procesos de aculturación, deriva en teorías y prácticas que contribuyen a mantener el status quo. La PL es un marco innovador para el análisis y la promoción del bienestar de las poblaciones inmigrantes en sociedades multiculturales justas (Fisher y Sonn, 2007; Smail, 2001) y permite proponer las siguientes recomendaciones (Hernández-Plaza et al., 2010): (1) tener en cuenta y conectar todos los niveles ecológicos involucrados en los problemas, para lo que es muy útil el trabajo interdisciplinario, (2) promover el empoderamiento individual y colectivo de los grupos desfavorecidos, y (3) utilizar la investigaciónacción participativa y el uso de las coaliciones comunitarias, para aumentar la aplicabilidad y la relevancia de los conocimientos adquiridos a la práctica real del colectivo inmigrante.

En este artículo se han señalado algunas herramientas útiles para que la población inmigrante resista y supere las condiciones de opresión, alcanzando así el bienestar y la integración. Una acción positiva es la promoción de organizaciones de base de inmigrantes como escenarios comunitarios empoderadores (Paloma et al., 2010). La participación en estas asociaciones promueve el desarrollo, fomenta el sentido de comunidad e identidad positiva, favorece la ayuda mutua y el desarrollo de redes de apoyo, aumenta el sentido de control y reduce la sensación de impotencia (Maton, 2008; Paloma et al., 2010; Wandersman y Florin, 2000). Por lo tanto, las organizaciones de base pueden desempeñar un papel clave en la promoción del bienestar y de cambios a nivel comunitario, relacional y personal. Al mismo tiempo, la población autóctona y sus instituciones tienen que ajustarse a la realidad social diversa. Destacamos la necesidad de promover Escenarios de Cuidados Multiculturales Empoderadores que promuevan competencia cultural entre sus profesionales y ayuden a transformar el entorno al que sirven. Las instituciones públicas también deben asegurar la accesibilidad y cercanía de los servicios comunitarios y el no aislamiento de individuos pertenecientes al mismo colectivo cultural en los vecindarios; así como promover una ideología multicultural y tolerante entre la población autóctona de las sociedades receptoras.

Finalmente, queremos resaltar el valor de formar coaliciones comunitarias para la investigación con poblaciones inmigrantes. Las iniciativas de investigación presentadas en este artículo han sido realizadas por una coalición compuesta por investigadores con diferentes perfiles (e.g., psicología comunitaria, psicología experimental, enfermería) y líderes comunitarios. Este trabajo colaborativo nos ha permitido acceder a herramientas de investigación muy diversas y a un conocimiento profundo de las comunidades. Estas coaliciones se componen habitualmente por investigadores, miembros clave de la comunidad y profesionales, que trabajan coordinadamente con el fin de llevar a cabo investigaciones en la comunidad orientadas a la comprensión, la denuncia y la transformación de situaciones de injusticia social. Desde estos escenarios se promueven y se apoyan acciones interdisciplinares que 
responden a las necesidades reales de las comunidades y son culturalmente sensibles.

En conclusión, se sugiere la incorporación de la perspectiva de la PL en las teorías sobre aculturación, así como en las agendas actuales sobre inmigración, con el objetivo de tener en cuenta la dinámica de poder en los diversos niveles ecológicos. La investigación y la acción social no están exentas de valores, por lo que proponemos que se tome el valor de la justicia social y la diversidad humana como condiciones necesarias para facilitar el bienestar personal, colectivo y comunitario de las poblaciones en desventaja.

\section{Referencias}

Albar, M.J. (2009). Poder y síndrome de burmout en enfermeras. Un modelo explicativo desde el enfoque psicopolítico. (Tesis doctoral no publlicada). Departamento de Psicología Social, Universidad de Sevilla.

Albar, M.J. y Bochinno, A. (2010) Taller de Capacitación en Competencia Cultural en Enfermeras de Urgencias Hospitalarias. Documento de trabajo interno de CESPYD, Universidad de Sevilla.

Balcázar, F.E., Garate-Serafini, T.J. y Keys, C.B. (2004). The need for action when conducting intervention research: The multiple roles of community psychologists. American Journal of Community Psychology, 33, 243-252.

Berry, J.W. (1997). Immigration, acculturation, and adaptation. Applied Psychology: An international review, 46 (1), 5-34.

Berry, J.W. (2005). Acculturation: living successfully in two cultures. International Journal of Intercultural Relations, 29, 697-712.

Berry, J.W. (2007). Acculturation strategias and adaptation. In J.E. Lansford, K. Deater-Deckard y M.H. Bornstein (Eds), Inmigrant Families in Contemporany Societies (pp. 69-82). New York: The Guilford Press.

Berry, J.W. (2008). Globalisation and acculturation. International Journal of Intercultural Relations, 32(4), 328-336.

Besabe, N., Páez, D., Aierdi, X., y JiménezAristizabal, A. (2009). Salud e inmigración. Aculturación, bienestar subjetivo y calidad de vida. Gipuzkoa: Observatorio Vasco de Inmigración.

Birman, D., Trickett, E. y Buchanan, R.M. (2005). A tale of two cities: Replication of a study on the acculturation and adaptation of immigrant adolescents from the Soviet Union in a Different Community Context. American Journal of Community Psychology, 35, 83-101.

Bourhis, R.Y., Moïse, L.C., Perreault, S., y Senécal, S. (1997). Towards an Interactive Acculturation Model: A social psychological approach. International Journal of Psychology, 32(6), 369-386.
Chirkov, V. (2009). Critical psychology of acculturation: What do we study and how do we study it, when we investigate acculturation? International Journal of Intercultural Relations, 33, 94-105.

Dana, R.H., y Allen, J.R. (2008). Cultural Competency Training in a Global Society. NY, USA: Springer

De la Mata, M.L., y Cubero, M. (2003). Psicología Cultural: aproximaciones al estudio de la relación entre mente y cultura. Infancia y Aprendizaje, 26(2), 181-199.

De la Mata, M.L., García-Ramírez, M., Santamaría, A. y Garrido, R. (2010). La integración de las personas migrantes: El enfoque de la Psicología Cultural y de la Liberación. En L. Melero (Ed.), Manual de intervención psicosocial con personas migrantes. Valencia: Fundación CeiMigra.

Dinh, K.T. y Bond, M.A. (2008). The other side of acculturation: Changes among host individuals and communities in their adaptation to immigrant populations. American Journal of Community Psychology, 46, 283-285

Domenig, D. (2008, November). The implementation of transcultural competence in the clinical context: Preconditions and challenges. Paper presented at the meeting of COST Action Home, Barcelona, Spain.

Fanon, F. (1963). The wretched of the Earth. New York: Grove Press.

Fisher, A.T. y Sonn, C.C. (2007). Power in community psychology research and practice. Journal of Community and Applied Social Psychology, 17, 255-257.

Fostier, J.P. (2010). Migrant-Sensitive Health Systems. OMS. Global Consultation on Migrant Health, National School of Public Health. Madrid, España

Freire, P. (1972). Pedagogía del oprimido. Buenos Aires, Argentina: Siglo XXI.

García-Ramírez, M. y Camacho, C. (2005). Adaptation y satisfaction among Moroccan in Spain: the community context role. Conference 40 years PostSwampscott: Community Psychology in Global Perspective. The University of Illinois at UrbanaChampaign, June, 9-12.

García-Ramírez, M., De la Mata, M., Paloma, V. y Hernández-Plaza, S. (en prensa). A liberation psychology approach to acculturative integration of migrant populations. American Journal of Community Psychology.

García-Ramírez, M., Martínez, M.F., Albar, M.J. y Santolaya, F.J. (2002). Inmigrantes y recursos sociales naturales. La aplicación del modelo del convoy social al proceso de aculturación. Migraciones, 11, $83-111$.

Garrido, R. (2010). El análisis de los vecindarios multiculturales como nuevos contextos de desarrollo. (Tesis de master no publicada). Departamento de Psicología Evolutiva y de la Educación, Universidad de Sevilla. 
Hernández-Plaza, S. (2003). La otra cara de la inmigración. Necesidades y sistemas de apoyo social. Almería, España: Servicio de Publicaciones de la Universidad de Almería.

Hernández-Plaza, S., Alonso, E. y Pozo, C. (2006). Social support interventions in migrant populations. British Journal of Social Work, 36, 1151-1169.

Hernández-Plaza, S., García-Ramírez, M., Camacho, C., y Paloma, V. (2010). New settlement and wellbeing in oppressive contexts: A liberation psychology approach. In C. Stuart (Ed.), The psychology of global mobility. International and Cultural Psychology Series (pp. 235-256). New York: Springer.

Hernández-Plaza, S., García-Ramírez, M., Herrera, I.M., Luque, V. y Paloma, V. (2009). Explorando poder, opresión y bienestar entre inmigrantes marroquíes en España. Proceedings of the II International Conference on Community Psychology. Lisboa, June 2008 .

Hernández-Plaza, S., Pozo, C. y Alonso, E. (2004). Apoyo social y bienestar subjetivo en un colectivo de inmigrantes marroquíes ¿Efectos directos o amortiguadores? Boletín de psicología, 80, 79-96.

Hernández-Plaza, S., Pozo, C., Alonso, E. y Martos, M.J. (2005). Estructura y funciones del apoyo social en un colectivo de inmigrantes marroquíes. Anales de Psicología, 21(2), 304-315.

Ingleby, D. (2006). Getting multicultural health care off the ground: Britain and the Netherlands compared. International Journal of Mental Health and Social Care, 2, 4-15.

Ingleby, M., Chimienti, P., Hatziprokopiou, M., Ormond, M. y De Freitas, C. (2005). The role of health in integration. En M.L. Fonseca y J. Malheiros (Eds.), Social integration and mobility (pp. 92-123). IMISCOE Cluster B5 State of the art report. Retrieved from http:// dare.uva.nl/document/21041.

Koehn, P.H. (2006). Globalization, migration health, and educational preparation for transnational medical encounters. Globalization and Health, 2(2), 1-16.

Luque-Ribelles, V. (2010). Condiciones Estructurales de Opresión. La visión de la comunidad inmigrante marroquí en Andalucía. Documento interno de CESPYD, Universidad de Sevilla.

MacMillan, D.W., y Chavis, D.M. (1986). Sense of community: A definition and theory. Journal of Community Psychology, 14(1), 6-23

Martín-Baró, I. (1986). Hacia una psicología de la liberación. Boletín de Psicología de El Salvador, 5 (22), 219-231.

Martín-Baró, I. (1994). Writings for a liberation psychology (Edited by A. Aron y S. Corne).Cambridge, MA: Harvard University Press.

Martínez, M. F., García-Ramírez, M., y Maya, I. (2001). Una tipología analítica de las redes de apoyo social de los inmigrantes africanos en Andalucía.
Revista Española de Investigaciones Sociológicas, 95, 99-125.

Martínez, M.F., García-Ramírez, M., Maya, I., Rodríguez, S. y Checa, F. (1996). La integración social de los inmigrantes africanos en Andalucía. Necesidades y recursos. Sevilla, España: Junta de Andalucía.

Maton, K.I. (2008). Empowering community settings: Agents of individual development, community betterment and positive social change. American Journal of Community Psychology, 41, 4-21.

Moane, G. (2003). Bridging the personal and the political: Practices for a liberation psychology. American Journal of Community Psychology, 31(1/2), 91-101.

Montero, M. y Sonn, C.C. (2009). About liberation and psychology: An introduction. In M. Montero y C.C. Sonn (Eds.), Psychology of liberation. Theory and applications (pp. 1-11). New York: Springer.

Navas, M., García, M.C., Sánchez, J., Rojas, A.J., Pumares, P. y Fernández, J.S. (2005). Relative Acculturation Model (RAEM): New contributions with regard to the study of acculturation. International Journal of Intercultural Relations, 29, 21-37.

Nelson, G. y Prilleltensky, I. (2005). The project of community psychology: Issues, values and tools for liberation and well-being. In G. Nelson y I. Prilleltensky (Eds.), Community Psychology. In pursuit of liberation and well-being (pp. 23-44). New York: Palgrave McMillan.

Oliveri, F. (2008). Policies for a fair multicultural society. On the use and abuse of "culture" in relation to migration issues. In Council of Europe, Reconciling migrants' well-being and the public interest Welfare state, firms and citizenship in transition (pp. 23-55). Belgium: Council of Europe Publishing.

Paloma-Castro, O. (2010). Análisis del hospital como promotor de comunidades multiculturales saludables. (Tesis de master no publicada). Departamento de Enfermería, Universidad de Cádiz.

Paloma, V., García-Ramírez, M., De la Mata, M. y Amal (2010). Acculturative-integration, self and citizenship construction: The experience of AmalAndaluza, a grassroots organization of Moroccan women in Andalusia. International Journal of Intercultural Relations, 34, 101-113.

Paloma, V., Herrera, I.M., y García-Ramírez, M. (2009). Psychopolitical validation of health promotion research for migrant populations: Conceptualizing well-being among Andalusian Moroccan immigrants. International Journal of Migration, Health and Social Care, 5(1), 25-33.

Penninx, R., Berger, M. y Kraal, K. (2006). The dynamics of international migration and settlement in Europe. A state of the art. Amsterdam: Amsterdam University Press.

Perkins, D. D., Florin, P., Rich, R. C., Wandersman, A., y Chavis, D. M. (1990). Participation and the social and physical environment of residential blocks: 
Crime and community context. American Journal of Community Psychology, 18, 83-116.

Perkins, D.D., y Taylor, R.B. (1996). Ecological assessments of community disorder: Their relationship to fear of crime and theoretical implications. American Journal of Community Psychology, 24 (1), 63-107.

Portes, A. y Rumbaut, R.G. (1996). Immigrant America: A portait (2nd ed.). Berkeley and Los Angeles: University of California Press.

Prilleltensky, I. (2008). Migrant well-being is a multilevel, dynamic, value dependent phenomenon. American Journal of Community Psychology, 42, 359-364.

Rudmin, F.W. (2006). Debate in science: the case of acculturation. In Anthro-Globe Journal. Retrieved from http://www.anthroglobe.ca/docs/ rudminf_ acculturation_061204.pdf

Rueda, J.F., y Navas, M.S. (1996). Hacia una evaluación de las nuevas formas

del prejuicio racial: las actitudes sutiles del racismo. Revista de Psicología Social, 11 (2), 131-149.

Sam, D.L. y Berry, J.W. (2006). The Cambridge handbook of acculturation psychology. Cambridge: Cambridge University Press.

Sevillano, J.M. (2010). Desarrollo de un instrumento para la evaluación de la competencia cultural comunitaria. (Tesis de master no publicada). Departamento de Psicología Experimental, Universidad de Sevilla.

Smail, D. (2001). De-psychologizing community psychology. Journal of Community and Applied Social Psychology, 11, 159-165.

Snowden, L.R. (2005). Racial, Cultural and Ethnic Disparities in Health and Mental Health: Toward Theory and Research at Community Levels. American Journal of Community Psycho-logy, $35(1 / 2), 1-8$.
Sonn, C.C., y Lewis, R.C. (2009). Immigration and Identity. The Ongoing Struggles for Liberation. In C.C. Sonn y M. Montero (Eds.), Psychology of Liberation. Theory and Applications (pp. 115- 134). New York, EEUU: Springer.

Soriano-Ayala, E. (Coord.). (2002). Interculturalidad: Fundamentos, programas y evaluación. Madrid, España: La Muralla, S.A.

Sue, D.W. (2001). Multidimensional facets of cultural competence. The Counseling Psychologist, 29, 790-821.

Sue, S., Zane, N., Nagayama Hall, G.C., y Berger, L.K. (2009). The Case for Cultural Competency in Psychotherapeutic Interventions. Annual Review Psychology, 60, 525-548.

Tseng, V. y Yoshikawa, H. (2008). Reconceptuali-zing acculturation: Ecological processes, historical contexts, and power inequities. American Journal of Community Psychology, 42, 355-358.

Wandersman, A. y Florin, P. (2000). Citizen participation and community organizations. In J. Rap-paport y E. Seidman (Eds.), Handbook of Community Psychology (pp. 247-272). New York: Kluwer Academic/Plenum Publishers.

Wandersman, A., y Nation, M. (1998). Urban neighborhoods and mental health: Psychological contributions to Understanding toxicity, resilience, and interventions. American Psychologist, 53(6), 647-656.

Ward, C. y Kagitcibasi, C. (2010). Acculturation theory, research and application: Working with and for communities. International Journal of Intercultural Relations, 34 (2), 97-190.

Ward, C. y Masgoret, A.M. (2006). A integrative model of attitudes toward immigrants. International Journal of Intercultural Relations, 30, 671-682.

Zimmerman, M.A. y Zahniser, J.H. (1991). Refinements of sphere-specific measures of perceived control: development of a sociopolitical control scale. Journal of Community Psychology, 19, 189-204.

Manuscrito Recibido: 19/05/2010

Revisión Recibida: 11/08/2010

Manuscrito Aceptado: 30/09/2010 